\title{
Convex Polytopes Whose Projection Bodies and Difference Sets Are Polars
}

\author{
Horst Martini \\ Sektion Mathematik, Pödagogische Hochschule, Wigardstrasse 17, \\ DDR-8060 Dresden, German Democratic Republic
}

\begin{abstract}
In a paper by the author and B. Weissbach it was proved that the projection body and the difference set of a $d$-simplex $(d \geq 2)$ are polars. Obviously, for $d=2$ a convex domain has this property if and only if its difference set is bounded by a so-called Radon curve. A natural question emerges about further classes of convex bodies in $R^{d}(d \geq 3)$ inducing the mentioned polarity. The aim of this paper is to show that a convex $d$-polytope $(d \geq 3)$ is a simplex if and only if its projection body and its difference set are polars.
\end{abstract}

\section{Definitions and Background Material}

Let $R^{d}(d \geqslant 3)$ denote the $d$-dimensional Euclidean vector space with scalar product $\langle\cdot, \cdot\rangle$, norm $\|\cdot\|$, and unit sphere $S^{d-1}:=\left\{u \in R^{d} \mid\langle u, u\rangle=1\right\}$. For basic notation the reader is referred to [4], [5], and [7]. In particular, we write $P^{d}$ for the set of convex $d$-polytopes, i.e., compact, convex subsets of $R^{d}$ with nonempty interior and a finite number of extreme points (vertices).

For $P \in P^{d}$ with $n \geq d+1$ facets let $a_{i}$ denote the outward normal vector of the $i$ th $(d-1)$-face, where $\left\|a_{t}\right\|$ represents the area of this facet $(i \in\{1, \ldots, n\})$. Then the vector sum of line segments given by

$$
\Pi P:=\sum_{i=1}^{n} \operatorname{conv}\left\{o, a_{t}\right\}=\frac{1}{2} \sum_{i=1}^{n} \operatorname{conv}\left\{-a_{i}, a_{t}\right\}
$$

is called the projection body of $P$ (see Section 7 in [4], and [11]). The polytope $\Pi P$ is a d-zonotope (see [9] and [11]) with center $o$, i.e., besides the body $\Pi P$ itself each face of it has a center of symmetry. Moreover, every segment summand conv $\left\{o, a_{i}\right\}(i=1, \ldots, n)$ generates a zone of facets in bd $\Pi P$, that is the intersection with the zonotope of supporting hyperplanes with normal vectors orthogonal 
to conv $\left\{o, a_{i}\right\}$. For that reason $\Pi P$ has exactly $m$ zones, where $m \leq n$ is the number of nonoriented facet normals of $P$. As is well known, a $(d-2)$-flat corresponds to each zone of the zonotope (and vice versa) in the so-called projective diagram $\bar{A}_{m}^{d-1}$ of $\Pi P$, which is a dissection of real projective $(d-1)$ space by $(d-2)$-flats with no common point into cells of various dimensions. More precisely, assuming these cells to be relatively bounded, every $(d-r-1)$ cell represents the intersection of $S^{d-1}$ and the closed normal cones of two opposite $r$-faces of $\Pi P(r=0, \ldots, d-1)$. Hence $\bar{A}_{m}^{d-1}$ is the projective representation of the usual spherical image (see Section I.5 of [1]) of the centrally symmetric polytope II $P$. Further, by

$$
(\Pi P)_{\delta}^{*}:=\left\{x \in R^{d} \mid\langle x, y\rangle \leq \delta^{2} \text { for all } y \in \Pi P\right\}
$$

the polar body of $\Pi P$ with respect to $\delta \cdot S^{d-1}\left(\delta \in R^{+}\right)$is defined. This polarity implies (see once more Section I.5 of [1]) that $\vec{A}_{m}^{d-1}$ coincides with the central projection of $(\Pi P)_{\delta}^{*}$, i.e., the projection of the $(d-2)$-skeleton of $(\Pi P)_{\delta}^{*}$ from the center $O$ onto the hyperplane at infinity belonging to the projective augmentation of $R^{d}$. (This way of projecting a polytope onto the real projective $(d-1)$-space is briefly discussed in Section 23 of [6]. Notice further that the $(d-2)$-skeleton of a polytope $P \in P^{d}$ is the union of its faces with dimensions not larger than $d-2$.)

The intersection of the $(d-2)$-skeleton of $P \in P^{d}$ and some hyperplane is called a $(d-2)$-circle of $P$, if it is homeomorphic to a $(d-2)$-sphere. A polytope $P \in P^{d}$ with center $o$ is said to be $(d-2)$-equatorial if, for each $(d-1)$-subspace $H$ through a $(d-2)$-face, the intersection of $H$ and the $(d-2)$-skeleton of $P$ is a $(d-2)$-circle. Moreover, such an intersection is called a $(d-2)$-equator of $P$. From the described correspondence between $\bar{A}_{m}^{d-1}$ and the central projection of $(\Pi P)_{\delta}^{*}$ it follows immediately that $(\Pi P)_{\delta}^{*}$ is a $(d-2)$-equatorial polytope. (Clearly, the $(d-2)$-equatoriality is only a necessary property of polars of $d$-zonotopes (see [2]). For necessary and sufficient conditions we can consult [14], whereas polars of $(d-2)$-equatorial polytopes are investigated in [3].)

Finally, the difference set of $P \in P^{d}$ is defined by

$$
\begin{aligned}
D P & :=P+(-1) P \\
& =\left\{x \in R^{d} \mid x=x_{1}-x_{2} \text { with } x_{1}, x_{2} \in P\right\}
\end{aligned}
$$

(see Section 7 of [4]). It should be noticed that we use $D M$ in an analogous manner if $M \subset R^{d}$ is an arbitrary point set.

\section{Proof of the Result}

We shall show the announced characteristic property of $d$-simplices $(d \geq 3)$ by proving the more general statement that a convex $d$-polytope $P$ is a simplex if and only if the central projection of $D P$ and the projective diagram of $\Pi P$ coincide. To see this, four lemmas are taken into consideration. 
Lemma 1. Let $P \in P^{d}(d \geq 3)$ be a polytope with coincidence between the central projection of DP and the projective diagram of $\Pi P$. Then $P$ is simplicial, i.e., each facet of the polytope is a $(d-1)$-simplex.

Proof. If $F(Q, u)$ is written for the intersection of a polytope $Q \in P^{d}$ and its supporting hyperplane with outward normal direction $u \in S^{d-1}$, then for $P \in P^{d}$ and each $u \in S^{d-1}$ the relation

$$
F(D P, u)=F(P, u)+F((-1) P, u)
$$

holds (see Section 15 of [5]). Further, it is known that every simplex (of dimension $d-1$ ) is indecomposable in the sense of vector addition, i.e., it has only positively homothetic summands [5, Section 15]. By the supposition of this lemma the central projection of $D P$ is a projective $(d-1)$-arrangement of $m(d-2)$-flats with no common point. As is shown in [12], such an arrangement has (at least) $m(d-1)$-cells which are projective simplices. Hence $D P$ has (at least) $2 m$ simplices as facets. By their indecomposability they cannot be vector sums of $r$-faces $(1 \leq r \leq d-2)$ of $P$ and $(-1) P$ in the sense of (4). Thus each of their $m$ nonoriented facet normals is also a facet normal of $P$, corresponding to simplex facets of this polytope in each case. Therefore nonsimplices as $(d-1)$-faces of $P$ are excluded.

Lemma 2. Let $P$ be a convex $d$-polytope $(d \geq 3)$. For every $(d-2)$-equator $\bar{M} \subset$ bd $D P$ there exists exactly one $(d-2)$-circle $M \subset \operatorname{bd} P$ with

$$
\left(x_{1}-x_{2}\right) \in \bar{M} \Rightarrow x_{1}, x_{2} \in M .
$$

Proof. For generating $\bar{M}$ it suffices to use the $(d-2)$-skeleton of $P$ (see (4) and the definition of $\bar{M}$ ). Let us consider the union of all difference sets generated from the intersections of this skeleton with hyperplanes whose normals are orthogonal to lin $\bar{M}$. Clearly, $\bar{M}$ is exactly the relative boundary of this union. We assume that more than one such difference set meets $\bar{M}$. Then there would exist a point $x \in \bar{M}$ belonging to the relative boundaries of two such difference sets $D P_{1}$ and $D P_{2}$, whose generators $P_{1}, P_{2} \subset P$ lie in different parallel hyperplanes. Hence

$$
x=x_{1}-x_{2}=y_{1}-y_{2}, \quad x_{1}, x_{2} \in P_{1}, \quad y_{1}, y_{2} \in P_{2},
$$

would hold. Since conv $\left\{x_{1}, x_{2}, y_{1}, y_{2}\right\}$ is a nondegenerate parallelogram, $x$ would be a relatively interior point of $\operatorname{conv}\left\{y_{1}-x_{2}, x_{1}-y_{2}\right\} \subset D P$.

By $\left(y_{1}-x_{2}\right),\left(x_{1}-y_{2}\right) \not \operatorname{lin} \bar{M}, x$ cannot lie in a face of $D P$ which fully belongs to $\operatorname{lin} \bar{M}$. But this contradicts the definition of $\bar{M}$. Thus $\bar{M}$ is generated by the difference set of exactly one linear $(d-1)$-cut of the used $(d-2)$-skeleton. The additivity of faces of convex bodies under vector addition (see (4)) implies that this linear cut is homeomorphic to a $(d-2)$-sphere, i.e., it is a $(d-2)$ - circle. 
Lemma 3. Let conv $M_{i}(i=1,2)$ be two $(d-1)$-simplices whose relative boundaries $M_{i}$ are $(d-2)$-circles of $P \in P^{d}$. If each $M_{i}$ generates $a(d-2)$-equator $\bar{M}_{i} \subset$ bd $D P$ (in the sense of the preceding lemma), then the simplices conv $M_{i}$ coincide in $d-1$ pairs of extreme points.

Proof. Since every edge of these simplices is also an edge of $P$, conv $M_{i}$ lies in a closed half-space with respect to aff $M_{j},\{i, j\}=\{1,2\}$. Let $v_{1}, v_{2} \in$ vert $P$ be two extreme points of conv $M_{2}$ with $v_{1}, v_{2} \notin$ conv $M_{1}$. We now distinguish two subcases regarding the relative position of conv $M_{1}$ and $\operatorname{conv}\left\{v_{1}, v_{2}\right\}$.

1. There exists no parallel projection of $R^{d}$ onto aff $M_{1}$ with the property that the image of conv $\left\{v_{1}, v_{2}\right\}$ is contained in conv $M_{1}$. Then we choose a parallel projection such that the image of $\operatorname{conv}\left\{v_{1}, v_{2}\right\}$ covers the longest chord of the same direction in conv $M_{1}$. The following considerations confirm the existence of such a projection.

For aff $\left\{v_{1}, v_{2}\right\} \|$ aff $M_{1}$ all possible image lines of aff $\left\{v_{1}, v_{2}\right\}$ in aff $M_{1}$ are parallel. Thus one of them contains the longest chord of conv $M_{1}$ with the same direction. By the supposition this chord is contained in an image of $\operatorname{conv}\left\{v_{1}, v_{2}\right\}$ on this line. For aff $\left\{v_{1}, v_{2}\right\} \nmid$ aff $M_{1}$ we use the point $\left\{p_{0}\right\}:=\operatorname{aff}\left\{v_{1}, v_{2}\right\} \cap$ aff $M_{1}$. If $\left\{v_{3}, \ldots, v_{d+2}\right\}$ denotes the vertex set of conv $M_{1}$, then

$$
p_{0}=\lambda_{3} v_{3}+\cdots+\lambda_{d+2} v_{d+2}, \quad \sum_{i=3}^{d+2} \lambda_{i}=1,
$$

is obtained. The assumed projection property implies $p_{0} \not$ conv $M_{1}$. Therefore at least one $\lambda_{i}$ above has to be negative and at least one positive. Without loss of generality we can write

$$
\lambda_{3}<0, \ldots, \lambda_{k}<0, \quad \lambda_{k+1} \geq 0, \ldots, \lambda_{d+2} \geq 0 \quad(3<k<d+2)
$$

and define

$$
\tau_{1}:=\sum_{i=3}^{k} \lambda_{i} \neq 0, \quad \tau_{2}:=\sum_{i=k+1}^{d+2} \lambda_{i} \neq 0, \quad \tau_{1}+\tau_{2}=1 .
$$

Introducing

$$
p_{1}:=\frac{1}{\tau_{1}} \sum_{i=3}^{k} \lambda_{i} v_{i} \text { and } p_{2}:=\frac{1}{\tau_{2}} \sum_{i=k+1}^{d+2} \lambda_{i} v_{i}
$$

we see by $p_{0}=\tau_{1} p_{1}+\tau_{2} p_{2}\left(\tau_{1}+\tau_{2}=1\right)$ that $p_{0}$ is a point from aff $\left\{p_{1}, p_{2}\right\}$. On the other hand, by (5) we have

$$
p_{1} \in \operatorname{conv}\left\{v_{3}, \ldots, v_{k}\right\}, \quad p_{2} \in \operatorname{conv}\left\{v_{k+1}, \ldots, v_{d+2}\right\}
$$

Hence

$$
\operatorname{aff}\left\{p_{1}, p_{2}\right\} \cap \operatorname{conv} M_{1}=\operatorname{conv}\left\{p_{1}, p_{2}\right\}
$$


Obviously, the sets $\left\{v_{3}, \ldots, v_{k}\right\}$ and $\left\{v_{k+1}, \ldots, v_{d+2}\right\}$ represent a dissection of ext conv $M_{1}$. Since conv $M_{1}$ is a $(d-1)$-simplex, there exist two uniquely determined parallel $(d-2)$ - flats $H_{1}, H_{2}$ in aff $M_{1}$ with

$$
\left\{v_{3}, \ldots, v_{k}\right\} \subset H_{1}, \quad\left\{v_{k+1}, \ldots, v_{d+2}\right\} \subset H_{2},
$$

and $p_{1} \in H_{1}, p_{2} \in H_{2}$. Because further conv $M_{1}$ is contained in $\operatorname{conv}\left(H_{1} \cup H_{2}\right)$, no chord of conv $M_{1}$ parallel to conv $\left\{p_{1}, p_{2}\right\}$ can be longer than this segment. Thus, the existence of a parallel projection with the demanded property is confirmed.

Now let us fix the position of the origin relative to conv $M_{1}$ and conv $\left\{v_{1}, v_{2}\right\}$. (Clearly, the presented relations are translation invariant. This determination of $o$ is only taken for the sake of convenience.) We denote by $v_{1}^{\prime}, v_{2}^{\prime}$ the images of $v_{1}, v_{2}$ with respect to the introduced parallel projection. Let $v_{1}^{\prime}$ coincide with $p_{1}$. Then $p_{2}$ (by the supposition a relatively interior point of $\operatorname{conv}\left\{v_{1}^{\prime}, v_{2}^{\prime}\right\}$ ) will be the origin.

Our next step is the confirmation of

$$
\operatorname{conv}\left\{v_{1}^{\prime}-\delta v_{2}^{\prime}, \delta v_{2}^{\prime}-v_{1}^{\prime}\right\} \subset D P
$$

for a suitable $\delta$ with $0<\delta<1$ (and $\delta \neq \frac{1}{2}$ ). To see this, we consider the parallelogram conv $\left\{v_{1}, v_{2}-v_{1}^{\prime},-v_{1}, v_{1}^{\prime}-v_{2}\right\}$. By (3) the vertices of this 4-gon belong to $D P$, and with $v_{1}, v_{2} \notin$ aff $M_{1}$ they do not lie in aff $M_{1}$.

We introduce a Cartesian coordinate system in the 2-plane of the parallelogram by using an $\bar{x}$ - and a $\bar{y}$-axis. By

$$
\begin{gathered}
v_{1}=\left(\bar{x}_{1}, \bar{y}_{1}\right), \quad \bar{x}_{1}, \bar{y}_{1}>0, \quad v_{1}^{\prime}=\left(\bar{x}_{1}, 0\right), \\
v_{2}=\left(-\bar{x}_{2}, \bar{y}_{2}\right), \quad \bar{x}_{2}, \bar{y}_{2}>0, \quad \bar{y}_{1} \neq \bar{y}_{2}, \quad v_{2}^{\prime}=\left(-\bar{x}_{2}, 0\right),
\end{gathered}
$$

the relations

$$
v_{2}-v_{1}^{\prime}=\left(-\bar{x}_{1}-\bar{x}_{2}, \bar{y}_{2}\right) \text { and }-v_{1}=\left(-\bar{x}_{1},-\bar{y}_{1}\right)
$$

hold. For $p=(\bar{x}, \bar{y})$ from aff $\left\{v_{2}-v_{1}^{\prime},-v_{1}\right\}$ the equation

$$
-\bar{x}_{2}\left(\bar{y}+\bar{y}_{1}\right)=\left(\bar{y}_{1}+\bar{y}_{2}\right)\left(\bar{x}+\bar{x}_{1}\right)
$$

is observed. The intersection of this line and the $\bar{x}$-axis is a point $q(\bar{q}, 0)$ with

$$
\bar{q}=-\bar{x}_{1}-\frac{\bar{y}_{1} \bar{x}_{2}}{\bar{y}_{1}+\bar{y}_{2}}, \quad \text { i.e., } \quad q=-v_{1}^{\prime}+\delta v_{2}^{\prime}, \quad \delta=\frac{\bar{y}_{1}}{\bar{y}_{1}+\bar{y}_{2}} \text {. }
$$

(Because $\bar{y}_{1}, \bar{y}_{2}>0$ the relation $0<\delta<1$ is clear, and $\bar{y} \neq \bar{y}_{2}$ implies $\delta \neq \frac{1}{2}$.)

Thus, by the symmetry of $D P$ with respect to $o$ the inclusion (6) is proved. Since $\left\|p_{1}-p_{2}\right\|$ is the maximal chord length of conv $M_{1}$ in the direction $v_{1}^{\prime} /\left\|v_{1}^{\prime}\right\| \in$ $S^{d-1}$, we have $\left(v_{1}^{\prime}-\delta v_{2}^{\prime}\right) \notin D M_{1}\left(D M_{1}=M_{1}+(-1) M_{1}\right)$, although the relation $\left(v_{1}^{\prime}-\delta v_{2}^{\prime}\right) \in\left(D P \cap\right.$ aff $\left.\bar{M}_{1}\right)$ holds. Thus, by Lemma 2 the $(d-2)$-circle $M_{1}$ cannot generate a $(d-2)$-equator of $D P$. 
2. There exists a parallel projection of the line segment $\operatorname{conv}\left\{v_{1}, v_{2}\right\}$ into the set conv $M_{1}$. We now use the oppositely oriented projection, namely from $\operatorname{conv}\left\{v_{1}^{\prime}, v_{2}^{\prime}\right\}$ onto $\operatorname{conv}\left\{v_{1}, v_{2}\right\}$. Since $\left\|v_{2}-v_{1}\right\|$ is an edge length of conv $M_{2}$, we get $\pm\left(v_{1}-v_{2}\right) \in$ ext $D M_{2}$ (see [8]). By (3) the points $\pm\left(v_{1}-v_{2}^{\prime}\right), \pm\left(v_{2}-v_{1}^{\prime}\right)$ belong to $D P$. On the other hand, the inclusions

$$
\pm\left(v_{1}-v_{2}\right) \in \text { relint conv }\left\{ \pm\left(v_{1}^{\prime}-v_{2}\right), \pm\left(v_{1}-v_{2}^{\prime}\right)\right\}
$$

hold. Since these line segments are not fully contained in aff $M_{2}$, the relative boundary of $M_{2}+(-1) M_{2}$ cannot be a $(d-2)$-equator of $D P$.

Lemma 4. For $P \in P^{3}$, let the central projection of $D P$ and the projective diagram $\bar{A}_{m}^{2}$ of $\Pi P$ coincide. Then $P$ is a tetrahedron.

Proof. Clearly, this coincidence implies that $P$ is simplicial (see Lemma 1 ) and that to each facet of $P$, there exists a parallel 1-equator in bd $D P$. Here we distinguish two subcases with respect to 1 -circles of $P$ that are generators of 1 -equators in bd $D P$.

1. Let us assume that every such 1 -circle is the relative boundary of a facet of $P$. Since $P$ has only triangles as facets, we can identify the sets conv $M_{l}$ and the points $v_{i}(i=1,2)$ from the proof of Lemma 3 with facets and vertices of $P$. Each nonsimplex $P \in P^{3}$ has two vertices not contained in one of its facets. (For the dual version of this statement see [13].) Thus, assuming that nonsimplices $P$ have no parallel 2 -faces, Lemma 3 implies the existence of facets of these polytopes not parallel to 1-equators in bd $D P$. On the other hand, if a nonsimplex $P \in P^{3}$ has a pair $\left\{\right.$ conv $M_{1}$, conv $\left.M_{2}\right\}$ of parallel 2 -faces, then by Lemma 2 (and suitable notation)

$$
\left(M_{1}+(-1) M_{1}\right) \subseteq \operatorname{relint}\left(M_{2}+(-1) M_{2}\right)
$$

must hold. Then let us consider the three facets having edges in common with conv $M_{1}$. Obviously, their longest chords in the directions of these edges are the edges themselves. Hence by (7) the relative boundaries of these three triangles cannot generate 1-equators in bd $D P$, too. We can continue this process of crossing over to further neighboring facets through all the boundary of $P$. But this contradicts the assumed existence of 2 -faces in bd $P$ whose relative boundaries generate 1 -equators in bd $D P$. Thus a nonsimplex $P \in P^{3}$ cannot have only relative facet boundaries as 1-circles which generate the 1-equators of $D P$.

2. Therefore we have to assume the existence of such a generating 1-circle $L \subset$ bd $P$ which is not a relative facet boundary of the nonsimplex $P \in P^{3}$. We denote by $\bar{G}$ the projective line representing $L+(-1) L$ in the central projection $\bar{A}_{m}^{2}$ of $D P$. Then each 0 -cell from $\bar{G}$ is contained in (at least) three lines of this projective 2-arrangement. Namely, by the connection of $\bar{A}_{m}^{2}$ with $\Pi P$ the line $\bar{G}$ must represent a zone of this zonohedron, i.e., a facet normal of $P$ which is orthogonal to aff $L$. Further, every edge of $P$ in $L$ belongs to two facets of the 
polyhedron whose normals are different and, additionally, different from the facet normal described above. Thus, arbitrarily oriented directions of these three normals form a linearly dependent system, whose representatives are pairwise linearly independent. Such a system corresponds to a triplet of projective lines through one 0 -cell from $\bar{G}$ in $\bar{A}_{m}^{2}$. In general, for a projective 2-arrangement $\bar{A}_{m}^{2}$ of $m$ lines with no common point the following relations are known or can be derived without difficulty (see Section 18 of [5]).

If $f_{i}(i \in\{0,1,2\})$ denotes the number of $i$-cells, $p_{,}(j \geq 3)$ the number of $j$-sided 2 -cells, and $w_{k}(k \geq 3)$ the number of 0 -cells which belong to $k$ projective lines of $\bar{A}_{m}^{2}$, then with $c:=\sum_{k \geq 3}(k-2) w_{k}$ we obtain $f_{0}-f_{1}+f_{2}=1$ (Euler's relation for $\left.\bar{A}_{m}^{2}\right), \sum_{j \geq 3} p_{j}=f_{2}, \sum_{j \geq 3} j p_{j}=2 f_{1}$, and $f_{1}=2 f_{0}+c$. It is obvious, that the first lemma implies $p_{3}=m$ and $p_{j}=0(j>4)$. A suitable combination of these equations leads to $2 c=m-4$. But since (at least) three projective lines of $\bar{A}_{m}^{2}$ are incident with every 0 -cell from $\bar{G}, 2 c$ cannot be smaller than $m-1$. Hence we finish, as in the first case, with a contradiction.

These lemmas allow the formulation of

Theorem 1. For a convex d-polytope $P(d \geq 3)$ the projective diagram of $\Pi P$ and the central projection of DP coincide if and only if $P$ is a simplex.

Proof. Because each simplex has this property (see [8]), only the converse implication remains to be verified. By means of Lemma 4 we may assume that in $P^{d-1}(d \geq 4)$ the simplices are characterized by such a coincidence. Further, we also assume that for $P \in P^{d}$ the projective arrangement $\bar{A}_{m}^{d-1}$ has this double meaning. We shall show that $P$ is then a $d$-simplex. Let $\bar{Q}$ be an arbitrary $(d-2)$-equator from bd $D P$. Obviously, the $(d-1)$-polytope conv $\bar{Q}$ is then $(d-3)$-equatorial. By Lemma 2 there exists a uniquely determined $(d-2)$-circle $Q \subset$ bd $P$ with conv $\bar{Q}=Q+(-1) Q$. We write $\bar{A}_{p}^{d-2}(p<m)$ for the relative central projection of $D Q:=Q+(-1) Q$ in the $(d-2)$-plane at infinity with respect to the projective augmentation of lin $D Q$. Now let us show that the assumed correspondence of $\bar{A}_{m}^{d-1}, \Pi P$, and $D P$ implies an analogous connection between $\bar{A}_{p}^{d-2}, D Q$, and the relative projection body $\Pi \hat{Q}$ of conv $Q=: \hat{Q}$. Clearly, an arbitrary relative facet of $\hat{Q}$ equals just a $(d-2)$-face of $P$. Thus such a face $R$ is the intersection of exactly two facets of $P$, whose normals span a 2 -subspace totally orthogonal to aff $R$. Therefore the set of the relative facet normals of $\hat{Q}$ is contained in the set of orthogonal images of facet normals of $P$ in lin $D Q$. If $q$ is the number of the relative facet normals of $\hat{Q}$, this means $q \leq p$. On the other hand, we have $p \leq q$. This follows by Shannon's result that $\bar{A}_{p}^{d-2}$ contains (at least) $p$ projective $(d-1)$-simplices, necessarily corresponding here to (at least) $p$ relative facet normals of $\hat{Q}$ (see Lemma 1). Hence $\Pi \hat{Q}$ and $D Q$ have the same $(d-2)$ arrangement as the relative projective diagram (resp. relative central projection). Hence, by the induction hypothesis $\hat{Q} \in P^{d-1}$ is a $(d-1)$-simplex.

Now tet $C$ denote the complex of all $(d-1)$-simplices $\hat{Q}_{i}$ which generate $(d-2)$-equators of $D P(i=1, \ldots, m)$. From a statement in [13] it follows that each nonsimplex $P \in P^{d}$ has a facet not containing two vertices of $P$. This and 
Lemma 3 imply that conv $C$ is a $d$-simplex, say $S$. If $\Pi P$ has less than $d+1$ zones, then $P$ is necessarily a $d$-parallelotope, whose difference set is not $(d-2)$-equatorial. Thus $m=d+1$ and $D S=D P$. Finally, we confirm that this equality implies $S=P$.

We have $S \subseteq P$. For $\left\{e_{0}, \ldots, e_{d}\right\}:=$ vert $S$ in [8] the relation

$$
\text { vert } D S=\left\{e_{i}-e_{j}\right\}, \quad i, j \in\{0, \ldots, d\}, \quad i \neq j,
$$

was shown. If there exists an extreme point $z$ of $P$ with $z \notin S$, then $\pm\left(z-z^{\prime}\right) \in$ vert $D S$ holds, where $z^{\prime}$ denotes a suitable point from vert $P$. For $z^{\prime} \in$ vert $S$ this relation contradicts (8), and $z^{\prime} \notin$ vert $S$ implies the existence of a pair $k, l$ from $\{0, \ldots, d\}$ with $\pm\left(e_{k}-e_{l}\right)= \pm\left(z-z^{\prime}\right)$. But then $e_{k}-e_{l}$ would be from the relative interior of conv $\left\{e_{k}-z^{\prime}, z-e_{l}\right\} \subset D P$, contradictory to $\left(e_{k}-e_{l}\right) \in$ vert $D P$.

\section{Formulation of the Result and Its Equivalents}

For assertions which are equivalent to the announced polarity property we introduce some additional notions. Let $V_{1}(P, u)$ denote the inner 1-quermass of $P \in P^{d}$ at $u \in S^{d-1}$, i.e., the length of the longest chord of this polytope in direction $u$. On the other hand, by $\vec{V}_{d-1}(P, u)$ we define the outer $(d-1)$-quermass (brightness) of $P$ at $u$, i.e., the area of the orthogonal image of $P$ in $\left\{x \in R^{d} \mid\langle x, u\rangle=0\right\}$ (see Section 7 of [4]). A (compact) $d$-prism $D_{u}$ with generators in direction $u$ is, by definition, the nondegenerate vector sum of a polytope from $P^{d-1}$ and a line segment with this direction. If under the condition $P \subseteq D_{u}$ the volume $V\left(D_{u}\right)$ is minimal, then this prism is said to be optimally circumscribed about $P \in P^{d}$. Now we are ready for

Theorem 2. The following properties of a convex $d$-polytope $P(d \geq 3)$ are equivalent:

(A) The polytope $P$ is a d-simplex.

(B) The difference set DP and the projection body $\Pi P$ are polars with respect to a sphere $\delta \cdot S^{d-1}$ for some $\delta \in R^{+}$.

(C) The inner 1 -quermass and the brightness of $P$ satisfy

$$
\underline{V}_{1}(P, u) \cdot \bar{V}_{d-1}(P, u)=\delta^{2}
$$

for each $u \in S^{d-1}$ and some $\delta \in R^{+}$.

(D) For $P$ all optimally circumscribed d-prisms have the same volume.

Proof. Since the introduced correspondence of $\Pi P, \bar{A}_{m}^{d-1}$, and $D P$ is a necessary condition for the polarity of $\Pi P$ and $D P$ with respect to $\delta \cdot S^{d-1}$, by [8] and the first theorem, $(\mathrm{A}) \Leftrightarrow(\mathrm{B})$ is confirmed. From the definitions of $\Pi P$ and $D P$ it follows that $V_{1}(P, u), u \in S^{d-1}$, is reciprocal to the restriction $g(D K, u):=$ $\min \{\rho>0 \mid u \in \rho D K\}$ to $S^{d-1}$ of the distance function of $D P$, whereas $\bar{V}_{d-1}(P, u)$ 
represents the correspondingly restricted support function $h(\Pi P, u):=$ $\max \{\langle u, y\rangle \mid y \in \Pi P\}$ of $\Pi P$. Since polarity of $\Pi P$ and $D P$ with respect to $\delta \cdot S^{d-1}$ $\left(\delta \in R^{+}\right)$is equivalent to $h(\Pi P, u) / g(D K, u)=\delta^{2}$ for all $u \in S^{d-1}$ [7, Section 12], (B) $\Leftrightarrow(C)$ is verified. Let $T_{u}$ denote a chord from $P \in P^{d}$ of maximal length in direction $u$. Then both endpoints of $T_{u}$ lie in parallel supporting hyperplanes of $P\left[4\right.$, Section 7]. Therefore $\min \left\{V\left(D_{u}\right) \mid P \subseteq D_{u}\right\}=\bar{V}_{d-1}(P, u) \cdot \underline{V}_{1}(P, u)$ for each $u \in S^{d-1}$ and $(C) \Leftrightarrow(D)$ are obtained.

\section{Concluding Remarks}

Obviously, by Theorem 2 the polarity of $\Pi P$ and the central symmetrization $\frac{1}{2} D P$ [7, Section 20], [4, Section 9] is also a characteristic property of simplices in $P^{d}$ $(d \geq 3)$. We might extend the investigations to the set $K^{d}$ of convex bodies (compact, convex sets with interior points) in $R^{d}$ and ask whether the polarity of $\Pi K$ and $D K$ implies that $K \in\left(K^{d} \backslash P^{d}\right)$ is a $d$-ellipsoid $(d \geq 3)$. For $d=2$, Theorem 2 remains true if $K^{d}$ is written instead of $P^{d}$ and if simplices are replaced by convex domains whose difference sets are bounded by Radon curves [7, Section 13]. Assuming polarity of $\Pi K$ and $D K$, we can estimate the constant $\delta^{2}$ (see Theorem 2) in terms of the volume $V(K)$ of $K \in K^{d}$ for $d \geq 2$. Namely, by $[8]$ and a result from $[10]$ we have

$$
\frac{2 \omega_{d-1}}{\omega_{d}} V(K) \leq \delta^{2} \leq d V(K), \quad \omega_{d}:=\frac{\pi^{d / 2}}{\Gamma(1+d / 2)}, \quad d \geq 2
$$

\section{References}

1. A. D. Aleksandrov, Konvexe Polyeder, Akademie-Verlag, Berlin, 1958.

2. E. D. Bolker, A class of convex bodies, Trans. Amer. Math. Soc. 145 (1969), 323-345.

3. E. D. Bolker, Centrally symmetric polytopes, in Proc. 12th Bienn. Internat. Seminar Canad. Math. Soc. Congr. (Vancouver, B.C., 1969), 255-263, Canadian Mathematical Society, Montreal, 1970.

4. T. Bonnesen and W. Fenchel, Theorie der konvexon Körper, Springer-Verlag, Berlin, 1974 (reprint).

5. B. Grünbaum, Convex Polytopes, Wiley, New York, 1967.

6. D. Hilbert and S. Cohn-Vossen, Anschauliche Geometrie, Springer-Verlag, Berlin, 1932.

7. K. Leichtweiss, Konvexe Mengen, Deutscher Verlag der Wissenschaften, Berlin, 1980.

8. H. Martini and B. Weissbach, On quermasses of simplices (submitted).

9. P. McMullen, On zonotopes, Trans. Amer. Math. Soc. 159 (1971), 91-109.

10. C. A. Rogers and G. C. Shephard, Some extremal problems for convex bodies, Mathematika 5 (1958), 93-102.

11. R. Schneider and W. Weil, Zonoids and related topics, in Convexity and Its Applications, edited by P. Gruber and J. M. Wills, 296-317, Birkhäuser-Verlag, Basel, 1983.

12. R. W. Shannon, Simplicial cells in arrangements of hyperplanes, Geom. Dedicata 8 (1979), 179-187.

13. H. Tverberg, How to cut a convex polytope into simplices? Geom. Dedicata 8 (1979), 239-240.

14. H. S. Witsenhausen, A support characterization of zonotopes, Mathematika 25 (1978), 13-16.

Received February 2, 1988, and in revised form June 9, 1988. 